\title{
EXCEPTIONAL VALUES OF ANALYTIC FUNCTIONS
}

\section{P. KRAJKIEWICZ}

\begin{abstract}
ABSTRACr. Let $g(z)$ be an analytic function with an isolated singularity at $z_{0}$ and let $f(z)$ be a nonanalytic multianalytic function with a nonessential isolated singularity at $z_{0}$. It is shown that if $g(z)-f(z)$ does not vanish in some deleted neighborhood of $z_{0}$, then $z_{0}$ is a nonessential singularity of $g(z)$.
\end{abstract}

Suppose that $g(z)$ is analytic with an isolated singularity at $z_{0}$, where $z_{0}$ is an arbitrary complex number, finite or infinite. Let $\bar{z}$ denote the complex conjugate of $z$. We offer the following result. If $g(z)-\bar{z}$ or $g(z)-1 / \bar{z}$ does not vanish in some deleted neighborhood of $z_{0}$, then $z_{0}$ is not an essential singularity of $g(z)$. This result follows from a characterization of bianalytic functions which do not vanish in some deleted neighborhood of an isolated singularity [2]. In case $g(z)$ is entire and $z_{0}=\infty$, the above result also follows from a theorem of M. B. Balk [1] which characterizes polyentire functions which do not vanish in some deleted neighborhood of $z_{0}=\infty$.

The foregoing result leads us to make the following definition. Let $z_{0}$ be an arbitrary complex number, finite or infinite. We define $E\left(z_{0}\right)$ to be the collection of all complex valued functions $f(z)$ defined in some deleted neighborhood of $z_{0}$ with the following property: If $g(z)$ is any analytic function with an isolated singularity at $z_{0}$ such that $g(z)-f(z)$ does not vanish in some deleted neighborhood of $z_{0}$, then $z_{0}$ is not an essential singularity of $g(z)$.

We are thus led to the problem of determining all the functions which belong to $E\left(z_{0}\right)$. We introduce a class of functions called multianalytic functions. It is shown that if $f(z)$ is a nonanalytic multianalytic function with a nonessential isolated singularity at $z_{0}$, then $f(z) \in E\left(z_{0}\right)$. If $g(z)$ is analytic with an isolated singularity at $z_{0}$, then as one application we obtain necessary and sufficient conditions in order that $(g(z))-\in E\left(z_{0}\right)$, where $(g(z))-$ denotes the complex conjugate of $g(z)$. As a further application we deduce the existence of fixed points for a certain class of analytic functions.

Received by the editors October 10, 1972 and, in revised form, December 26, 1972. AMS (MOS) subject classifications (1970). Primary 30A68; Secondary 30A78, 30A96.

Key words and phrases. Exceptional values, analytic functions, multianalytic functions, fixed points, quasinormal families. 
We now introduce some notation and give some definitions. Let $z_{0}$ be an arbitrary complex number, finite or infinite, and let $0<R<+\infty$. If $z_{0}$ is finite, then $A\left(z_{0}, R\right)$ will denote the set of all finite complex numbers $z$ such that $0<\left|z-z_{0}\right|<R$. If $z_{0}=\infty$, then $A\left(z_{0}, R\right)$ will denote the set of all finite complex numbers $z$ such that $R<|z|<+\infty$. We also let $N\left(z_{0}, R\right)=$ $A\left(z_{0}, R\right) \cup\left\{z_{0}\right\}$.

A function $f(z)$ defined on $A\left(z_{0}, R\right)$ is termed multianalytic on $A\left(z_{0}, R\right)$ if and only if there is some nonnegative integer $n$ and a sequence $f_{k}(z)$ of functions analytic on $A\left(z_{0}, R\right)$ for $k \geqq-n$ such that

$$
f(z)=\sum_{k=-n}^{+\infty}\left(\left(z-z_{0}\right)-\right)^{k} f_{k}(z),
$$

for all $z$ in $A\left(z_{0}, R\right)$, where $\left(z-z_{0}\right)$ - denotes the complex conjugate of $\left(z-z_{0}\right)$. If $z_{0}=\infty$ then in the series in equation (1) it is understood that the term $\left(\left(z-z_{0}\right)^{-}\right)^{k}$ is replaced by $1 / \bar{z}^{k}$ for each $k \geqq-n$. It is also understood that the series in equation (1) is almost uniformly convergent on $A\left(z_{0}, R\right)$, that is the series is uniformly convergent on every nonempty closed subset of $A\left(z_{0}, R\right)$. If $z_{0}$ is any complex number, finite or infinite, then a function $f(z)$ is said to be multianalytic at $z_{0}$ if and only if there is some $0<R<+\infty$ so that $f(z)$ is multianalytic on $A\left(z_{0}, R\right)$.

Next let $f(z)$ be multianalytic on $A\left(z_{0}, R\right)$ and represented on $A\left(z_{0}, R\right)$ by equation (1). We shall show later that the functions $f_{k}(z)$ in equation (1) are uniquely determined on $A\left(z_{0}, R\right)$ by $f(z)$. For each $k \geqq-n$, we define $d_{k}=d\left(f_{k}\right)$, the order of $f_{k}(z)$ at $z_{0}$, as follows. If $f_{k}(z) \equiv 0$ on $A\left(z_{0}, R\right)$, then $d_{k}=-\infty$. If $z_{0}$ is an essential singularity of $f_{k}(z)$, then $d_{k}=+\infty$. Now suppose that $f_{k}(z) \not \equiv 0$ on $A\left(z_{0}, R\right)$ and that $z_{0}$ is a nonessential singularity of $f_{k}(z)$. If $z_{0} \neq \infty$, then $d_{k}$ is that unique integer such that $\left(z-z_{0}\right)^{d_{k}} f_{k}(z)$ is analytic and not zero at $z_{0}$. If $z_{0}=\infty$, then $d_{k}$ is that unique integer such that $f_{k}(z) / z^{d_{k}}$ is analytic and not zero at $z_{0}=\infty$. We then define the order $d$ of $f(z)$ at $z_{0}$ to be the least upper bound of the numbers $d_{k}-k$ for $k \geqq-n$. Note that $d$ is an integer or $\pm \infty$. Observe that $d=-\infty$ if and only if $f(z) \equiv 0$ on $A\left(z_{0}, R\right)$. The point $z_{0}$ is termed an essential or a nonessential singularity of $f(z)$ according as $d=+\infty$ or $d \neq+\infty$.

Note that if $f(z)$ is multianalytic at $z_{0}$ of order $d$ and if $h(z)$ is a Möbius transformation, then $f[h(z)]$ is multianalytic at $h^{-1}\left(z_{0}\right)$ of the same order $d$.

We now need some preliminary lemmas.

LEMMA 1. Let $f(z)$ be multianalytic on $A(\infty, R)$ and represented on $A(\infty, R)$ by equation (1) where $z_{0}=\infty$. Then for $\rho>R$, the series

$$
f(z, \rho)=\sum_{k=-n}^{+\infty} \frac{z^{k} f_{k}(z)}{\rho^{2 k}},
$$

is almost uniformly convergent on $R<|z| \leqq \rho$. 
Proof. Observe that the general term of the series in equation (2) can be written in the form $z^{k} f_{k}(z) / \rho^{2 k}=\left(z \bar{z} / \rho^{2}\right)^{k} f_{k}(z) / \bar{z}^{k}$. For $k \geqq 0$, the sequence $\left(z \bar{z} / \rho^{2}\right)^{k}$ is decreasing and uniformly bounded for $R<|z| \leqq \rho$. Hence by Abel's theorem the series in equation (2) is almost uniformly convergent in $R<|z| \leqq \rho$ as claimed.

Note that the auxiliary function $f(z, \rho)$ defined by equation (2) is continuous on $R<|z| \leqq \rho$ and analytic on $R<|z|<\rho$ and that $f(z, \rho)=f(z)$ for $|z|=\rho>R$.

We now have the following uniqueness result to which we appealed earlier.

LEMMA 2. Let $f(z)$ be multianalytic on $A\left(z_{0}, R\right)$ and represented on $A\left(z_{0}, R\right)$ by equation (1). Then the functions $f_{k}(z)$ in equation (1) are uniquely determined on $A\left(z_{0}, R\right)$ by $f(z)$.

Proof. It suffices to show that if $f(z) \equiv 0$ on $A\left(z_{0}, R\right)$, then $f_{k}(z) \equiv 0$ on $A\left(z_{0}, R\right)$ for each $k \geqq-n$. We need only consider the case when $z_{0}=\infty$. Let $f(z)$ be represented on $A(\infty, R)$ by equation (1) where $z_{0}=\infty$. From equation (2) we see that $f(z, \rho)=0$ for $|z|=\rho>R$. Hence $f(z, \rho)=0$ for $R<|z| \leqq \rho$. Let $z \in A(\infty, R)$ be fixed. From Lemma 1 , it follows that the Laurent series $g(w)=\sum z^{k} f_{k}(z) / w^{2 k}, k \geqq-n$, is convergent in case $w$ is real and $w>|z|$. Hence the Laurent series $g(w)$ is almost uniformly convergent on $A(\infty,|z|)$. Also $g(w)=0$ in case $w$ is real and $w>|z|$. Hence $g(w) \equiv 0$ on $A(\infty,|z|)$ by the principle of permanence. From the uniqueness of the Laurent series expansion for $g(w)$ it follows that $z^{k} f_{k}(z)=0$ for $k \geqq-n$. Since $z$ is an arbitrary point of $A(\infty, R)$, it follows that $z^{k} f_{k}(z) \equiv 0$ on $A(\infty, R)$ for each $k \geqq-n$ and the result follows.

We now need two technical lemmas regarding the auxiliary function $f(z, \rho)$.

LEMMA 3. Let $f(z)$ be multianalytic on $A(\infty, R)$ and represented on $A(\infty, R)$ by equation (1) where $z_{0}=\infty$. Assume that $f_{0}(z) \equiv 0$ on $A(\infty, R)$ and that $f(z)$ is of order $d \neq \pm \infty$ at $z_{0}=\infty$. Then there exists a function $P(z)$ analytic and not identically constant on $A(0,1)$ such that $f(\rho z, \rho) / \rho^{d} z^{d} \rightarrow$ $P(z)$ as $\rho \rightarrow+\infty$ almost uniformly in $A(0,1)$.

Proof. If $d_{k} \neq-\infty$, then $f_{k}(z) / z^{d_{k}}$ is analytic and not zero at $z_{0}=\infty$. In this case let $a_{k} \neq 0$ be the value of $f_{k}(z) / z^{d_{k}}$ at $z_{0}=\infty$. Clearly $d_{k} \neq-\infty$ for at least one $k \geqq-n$. Now

$$
\frac{f(\rho z, \rho)}{\rho^{d} z^{d}}=\sum z^{2 k}(\rho z)^{d_{k-k-d}} \frac{f_{k}(\rho z)}{(\rho z)^{d_{k}}}, \quad k \geqq-n, d_{k} \neq-\infty,
$$

for $R / \rho<|z|<1$. From the above equation it seems reasonable to suppose that $f(\rho z, \rho) \rho^{d} z^{d} \rightarrow \sum a_{k} z^{2 k}, k \geqq-n, d_{k}-k=d$, almost uniformly with 
respect to $z$ in $A(0,1)$ as $\rho \rightarrow+\infty$. We shall show that the above result is true. Let $\sigma>R$ be fixed. Since the series in equation (1) is uniformly convergent on $|z|=\sigma$, then there is some positive constant $M$ so that $\left|f_{k}(z)\right| z^{k} \mid \leqq$ $M$ for $|z|=\sigma$ and $k \geqq-n$. Hence $\left|f_{k}(z) / z^{d_{k}}\right| \leqq M / \sigma^{d_{k}-k}$ for $|z|=\sigma$ when $d_{k} \neq$ $-\infty$. Since $f_{k}(z) / z^{d_{k}}$ is analytic on $A(\infty, R)$ then by the maximum modulus principle we see that $\left|f_{k}(z)\right| z^{d_{k}} \mid \leqq M / \sigma^{d_{k}-k}$ for $\sigma \leqq|z| \leqq+\infty$ whenever $d_{k} \neq$ $-\infty$. Hence $\left|a_{k}\right| \leqq M / \sigma^{d_{k}-k}$ when $d_{k} \neq-\infty$. Thus $\left|a_{k}\right| \leqq M / \sigma^{d}$ when $d_{k}-$ $k=d$. Thus the function $P(z)=\sum a_{k} z^{2 k}, k \geqq-n, d_{k}-k=d$, is analytic and not identically constant in $A(0,1)$. We can now write $f(\rho z, \rho) / \rho^{d} z^{d}-$ $P(z)=\sum_{1} z^{2 k}(\rho z)^{d_{k}-k-d} f_{k}(\rho z)^{d_{k}} /(\rho z)^{d_{k}}+\sum_{2} z^{2 k}\left(f_{k}(\rho z) /(\rho z)^{d_{k}}-a_{k}\right)$, for $R / \rho<$ $|z|<1$, where in the first series $\sum_{1}$ we sum over all $k \geqq-n$ such that $d_{k} \neq$ $-\infty$ and $d_{k}-k \leqq d-1$ and in the second series $\sum_{2}$ we sum over all $k \geqq-n$ such that $d_{k}-k=d$. We now proceed to obtain upper bounds for the two series $\sum_{1}$ and $\sum_{2}$. First note that $\left|f_{k}(\rho z)^{d_{k}}\right| \leqq M / \sigma^{d_{k}-k}$ for $\sigma \leqq \rho|z| \leqq+\infty$ when $d_{k} \neq-\infty$. Thus if $\sigma / \rho<|z|<1$ we see that

$$
\begin{aligned}
\left|\sum_{1}\right| & \leqq \sum_{1}|z|^{2 k}|\rho z|^{d_{k-k-d}} M / \sigma^{d k-k}=M \sigma^{-d} \sum_{1}|z|^{2 k}|\sigma / \rho z|^{d-\left(d_{k}-k\right)} \\
& \leqq M \sigma^{-d} \sum_{1}|z|^{2 k}|\sigma / \rho z| \leqq M|z|^{-2 n-1} / \rho \sigma^{d-1}\left(1-|z|^{2}\right) .
\end{aligned}
$$

Next note that $\left|f_{k}(z) / z^{d_{k}}-a_{k}\right| \leqq 2 M / \sigma^{d_{k}-k}$ for $\sigma \leqq|z| \leqq+\infty$ when $d_{k} \neq-\infty$. Now the function $f_{k}(z) / z^{d_{k}}-a_{k}$ is analytic on $A(\infty, R)$ and vanishes at $z_{0}=\infty$. Hence by Schwarz's lemma we deduce that $\left|f_{k}(z) / z^{d_{k}}-a_{k}\right| \leqq$ $2 M /|z| \sigma^{d_{k}-k-1}$ for $\sigma \leqq|z| \leqq+\infty$ when $d_{k} \neq-\infty$. Hence

$$
\left|f_{k}(\rho z)^{d_{k}} /(\rho z)^{d_{k}}-a_{k}\right| \leqq 2 M / \rho|z| \sigma^{d-1} \text { for } \sigma \leqq \rho|z| \leqq+\infty
$$

when $d_{k}-k=d$. Thus if $\sigma / \rho<|z|<1$ we see that

$$
\left|\sum_{2}\right| \leqq \sum_{2}|z|^{2 k} 2 M / \rho|z| \sigma^{d-1} \leqq 2 M|z|^{-2 n-1} / \rho \sigma^{d-1}\left(1-|z|^{2}\right) .
$$

From the foregoing upper bounds for $\Sigma_{1}$ and $\sum_{2}$ we deduce that

$$
\left|f(\rho z, \rho) / \rho^{d} z^{d}-P(z)\right| \leqq 3 M|z|^{-2 n-1} / \rho \sigma^{d-1}\left(1-|z|^{2}\right)
$$

for $\sigma / \rho<|z|<1$. The result now follows.

Lemma 4. Let $f(z)$ be multianalytic on $A(\infty, R)$ and of order $d \neq$ $\pm \infty$ at $z_{0}=\infty$. Then there is an integer $m$ and a function $h(z)$ analytic and not identically zero on $A(\infty, R)$ with a nonessential singularity at $z_{0}=\infty$ such that $\rho^{m} f(z, \rho) \rightarrow h(z)$ as $\rho \rightarrow+\infty$ almost uniformly on $A(\infty, R)$.

Proof. Let $f(z)$ be represented on $A(\infty, R)$ by equation (1) where $z_{0}=\infty$. There is some integer $s \geqq-n$ so that $f_{k}(z) \equiv 0$ on $A(\infty, R)$ for $-n \leqq$ 
$k \leqq s-1$ but $f_{s}(z) \not \equiv 0$ on $A(\infty, R)$. Hence $\rho^{2 s} f(z, \rho)=\sum z^{k} f_{k}(z) / \rho^{2(k-s)}$, $k \geqq s$, for $R<|z| \leqq \rho$. From the above equation it seems reasonable to suppose that $\rho^{2 s} f(z, \rho) \rightarrow z^{s} f_{s}(z)$ as $\rho \rightarrow+\infty$ almost uniformly on $A(\infty, R)$. We shall show that this is the case. Let $\sigma>R$ be fixed. Since the series in equation (1) is uniformly convergent on $|z|=\sigma$, there is some $M>0$ so that $\left|f_{k}(z) / z^{k}\right| \leqq M$ for $|z|=\sigma$ and $k \geqq s$. Hence $\left|f_{k}(z) / z^{d_{k}}\right| \leqq M / \sigma^{d_{k}-k}$ for $|z|=\sigma$ and $k \geqq s$ when $d_{k} \neq-\infty$. Now $f_{k}(z) / z^{d_{k}}$ is analytic on $A(\infty, R)$ so that by the maximum modulus principle we deduce that $\left|f_{k}(z)\right| z^{d_{k}}|\leqq M| \sigma^{d_{k}-k}$ for $\sigma \leqq|z| \leqq+\infty$ and $k \geqq s$ when $d_{k} \neq-\infty$. Thus if $k \geqq s$ and $d_{k} \neq-\infty$ and $\sigma \leqq|z|<+\infty$ we see that

$$
\begin{aligned}
\left|z^{k} f_{k}(z)\right| & =\left|z^{d_{k}+k} f_{k}(z) / z^{d_{k}}\right| \leqq|z|^{d_{k}+k} M / \dot{\sigma}^{d_{k}-k} \\
& =M|z|^{2 k}|z / \sigma|^{d_{k}-k} \leqq M|z|^{2 k}|z / \sigma|^{d}=M|z|^{d+2 k} / \sigma^{d} .
\end{aligned}
$$

Thus for all $k \geqq s$ we see that $\left|z^{k} f_{k}(z)\right| \leqq M|z|^{d+2 k} / \sigma^{d}$ when $\sigma \leqq|z|<+\infty$. From the above estimate it is now easy to see that $\left|\rho^{2 s} f(z, \rho)-z^{s} f_{s}(z)\right| \leqq$ $M|z|^{d+2 s+2} / \sigma^{d}\left(\rho^{2}-|z|^{2}\right)$ for $R<\sigma \leqq|z|<\rho$. The result now follows.

If $f(z)$ is an arbitrary complex valued function which is continuous and never zero on $|z|=\rho>0$, we define $\Delta_{\rho} f(z)$ to be $1 / 2 \pi$ times the change in the argument of $f(z)$ around the positively oriented circumference $|z|=\rho$.

We are now in a position to establish our main result.

THEOREM. Let $f(z)$ be multianalytic and not analytic on $A\left(z_{0}, R\right)$ with a nonessential singularity at $z_{0}$. Then $f(z) \in E\left(z_{0}\right)$.

Proof. We need only consider the case when $z_{0}=\infty$. Let $f(z)$ be multianalytic and not analytic on $A(\infty, R)$ and of order $d \neq \pm \infty$ at $z_{0}=\infty$ and let $g(z)$ be analytic on $A(\infty, R)$ such that $g(z)-f(z)$ does not vanish on $A(\infty, R)$. We wish to show that $z_{0}=\infty$ is not an essential singularity of $g(z)$. We shall suppose that $z_{0}=\infty$ is an essential singularity of $g(z)$ and arrive at a contradiction. We need only consider the case when $d=0$. Further if $f(z)$ is represented on $A(\infty, R)$ by equation (1) where $z_{0}=\infty$, we need only consider the case when $f_{0}(z) \equiv 0$ on $A(\infty, R)$. There is some integer $p$ so that $\Delta_{\rho}[g(z)-f(z)]=p$ for all $\rho>R$. Hence $\Delta_{\rho}[g(z)-f(z, \rho)]=p$ for all $\rho>R$. From Lemma 4 , there is an integer $m$ and a function $h(z)$ analytic and not identically zero on $A(\infty, R)$ with a nonessential singularity at $z_{0}=\infty$ such that $\rho^{m} f(z, \rho) \rightarrow h(z)$ as $\rho \rightarrow+\infty$ almost uniformly on $A(\infty, R)$. Now choose some fixed $\sigma>R$ so that $g(z) \neq 0$ and $h(z) \neq 0$ and $g(z) \neq h(z)$ for all $z$ on $|z|=\sigma$. Now $\rho^{m} g(z)-\rho^{m} f(z, \rho) \rightarrow \infty$ or $g(z)-h(z)$ or $-h(z)$ as $\rho \rightarrow+\infty$ uniformly on $|z|=\sigma$ according as $m \geqq 1$ or $m=0$ or $m \leqq-1$. It is easy to see that there is some integer $q$ and some $\lambda>\sigma$ so that $\Delta_{\sigma}[g(z)-f(z, \rho)]=q$ for all $\rho>\lambda$. We thus see that the equation $g(z)=$ $f(z, \rho)$ has exactly $p-q=r$ solutions, counting multiplicities, in $\sigma<|z|<\rho$ 
for all $\rho>\lambda$. It follows that each of the equations $g(\rho z)=f(\rho z, \rho)$ and $g(\rho z)=f(\rho z, 2 \rho)$ has at most $r$ solutions in $\sigma|\lambda<| z \mid<1$ for all $\rho>\lambda$. From Lemma 3 , there is a function $P(z)$ analytic and not constant in $A(0,1)$ such that $f(\rho z, \rho) \rightarrow P(z)$ as $\rho \rightarrow+\infty$ almost uniformly on $A(0,1)$. Now $P(z / 2)-P(z) \not \equiv 0$ on $A(0,1)$. Hence there is some $\sigma / \lambda<a<b<1$ so that $P(z) \neq 0$ and $P(z / 2)-P(z) \neq 0$ for $a \leqq|z| \leqq b$. Now $f(\rho z, 2 \rho)-f(\rho z, \rho) \rightarrow$ $P(z / 2)-P(z)$ as $\rho \rightarrow+\infty$ almost uniformly on $A(0,1)$. Hence there is some $\mu>\lambda$ so that $f(\rho z, 2 \rho)-f(\rho z, \rho) \neq 0$ and $f(\rho z, \rho) \neq 0$ for $a \leqq|z| \leqq b$ and $\rho>\mu$. Hence we can find positive constants $A, B$, and $C$ so that $A>$ $B|f(\rho z, 2 \rho)-f(\rho z, \rho)|>2|f(\rho z, \rho)|>2 C$ for $a \leqq|z| \leqq b$ and $\rho>\mu$. We shall utilize these inequalities later in the proof. Thus for $\rho>\mu$, the function

$$
[g(\rho z)-f(\rho z, \rho)] /[f(\rho z, 2 \rho)-f(\rho z, \rho)]
$$

is analytic on $a<|z|<b$ and assumes each of the values zero and one at most $r$ times in $a<|z|<b$. Let $H$ denote the collection of these functions for $\rho>\mu$. This family $H$ is a family of functions which is analytic on $a<|z|<b$ and quasinormal on $a<|z|<b$ of order not exceeding $r$ [3]. Hence there is some $a<c<b$ and some sequence $\rho_{n}>\mu$ diverging to $+\infty$ so that the sequence

$$
h_{n}(z)=[g(\rho z)-f(\rho z, \rho)] /[f(\rho z, 2 \rho)-f(\rho z, \rho)] \text { for } \rho=\rho_{n}
$$

is either uniformly convergent on $|z|=c$ or is uniformly divergent to $\infty$ on $|z|=c$. In either case we shall arrive at a contradiction by showing that $z_{0}=\infty$ is not an essential singularity of $g(z)$. First suppose that the sequence $h_{n}(z)$ is uniformly convergent on $|z|=c$. Hence there is some $K>0$ so that $\left|h_{n}(z)\right| \leqq K$ for $|z|=c$. Hence $|g(z)|<K(A / 2+A / B)$ for $|z|=c \rho_{n}$. Hence $z_{0}=\infty$ is not an essential singularity of $g(z)$. Next suppose that the sequence $h_{n}(z)$ is uniformly divergent to $\infty$ on $|z|=c$. Hence there is some integer $n_{0}$ so that $\left|h_{n}(z)\right|>B$ for $|z|=c$ and $n \geqq n_{0}$. Thus

$$
|g(\rho z)-f(\rho z, \rho)|>B|f(\rho z, 2 \rho)-f(\rho z, \rho)|>2|f(\rho z, \rho)|>2 C
$$

for $|z|=c$ and $\rho=\rho_{n}$ and $n \geqq n_{0}$. From the above inequalities we make two deductions. First it follows that $|g(z)|>C$ for $|z|=c \rho_{n}$ and $n \geqq n_{0}$. Second it follows that $\Delta_{c} g(\rho z)=\Delta_{c}[g(\rho z)-f(\rho z, \rho)] \leqq p$ for $\rho=\rho_{n}$ and $n \geqq n_{0}$ so that $g(z)$ does not vanish in some deleted neighborhood of $z_{0}=\infty$. Hence $g(z)$ does not have an essential singularity at $z_{0}=\infty$. This completes the proof of the theorem.

As immediate consequences of the above theorem we offer the following corollaries.

COROllary 1. Let $g(z)$ be nonconstant and analytic on $A\left(z_{0}, R\right)$. Then $(g(z))-\in E\left(z_{0}\right)$ if and only if $z_{0}$ is a nonessential singularity of $g(z)$. 
Proof. If $z_{0}$ is a nonessential singularity of $g(z)$, then from the above theorem we have that $(g(z))-\in E\left(z_{0}\right)$. Next suppose that $z_{0}$ is an essential singularity of $g(z)$. Let $f(z)=g(z)+1$ for $z \in A\left(z_{0}, R\right)$. Then $f(z)$ is analytic on $A\left(z_{0}, R\right)$ with an essential singularity at $z_{0}$. Clearly $f(z)-(g(z))-$ does not vanish on $A\left(z_{0}, R\right)$. Hence $(g(z))-\notin E\left(z_{0}\right)$. This proves the corollary.

COROLlaRy 2. Let $g_{1}(z), \cdots, g_{n}(z)$ and $h_{1}(z), \cdots, h_{n}(z)$ be analytic on $A\left(z_{0}, R\right)$ with a nonessential singularity at $z_{0}$. If $f(z)=g_{1}(z)\left(h_{1}(z)\right)-+$ $\cdots+g_{n}(z)\left(h_{n}(z)\right)$ - is not analytic on $A\left(z_{0}, R\right)$, then $f(z) \in E\left(z_{0}\right)$.

COROLLARY 3. Let $z_{0} \neq \infty$ and $R>0$ and let $a_{r s}$ be a sequence of complex numbers for $r, s \geqq 0$. If $f(z)=\sum a_{r s}\left(z-z_{0}\right)^{r}\left(\left(z-z_{0}\right)-\right)^{s}$ is defined and not analytic in $\left|z-z_{0}\right|<R$, then $f(z) \in E\left(z_{0}\right)$.

In [4], P. C. Rosenbloom showed that every entire transcendental function possesses fixed points of exact order one or two. We obtain an extension of the above result to a certain class of analytic functions.

COROllary 4. Let $g(z)$ be analytic on $A(\infty, R)$ with an essential singularity at $z_{0}=\infty$ and suppose that $g(z)$ is real valued on the real axis. Then $g(z)$ possesses a fixed point of exact order one or two in $A(\infty, R)$.

Proof. First $(g(z))-=g(\bar{z})$ for all $z$ in $A(\infty, R)$. From Corollary 1 , there is some $\zeta$ in $A(\infty, R)$ so that $g(\zeta)=\bar{\zeta}$. Hence $g(\bar{\zeta})=\zeta$. Hence $g[g(\bar{\zeta})]$ is defined and $g[g(\bar{\zeta})]=\bar{\zeta}$ so that $\bar{\zeta}$ is a fixed point of $g(z)$ of exact order one or two in $A(\infty, R)$.

\section{REFERENCES}

1. M. B. Balk, Entire polyanalytic functions with a bounded set of zeros, Izv. Akad. Nauk Armjan. SSR Ser. Mat. 1 (1966), no. 5, 341-357. MR 34 \#6127.

2. P. Krajkiewicz, Bianalytic functions with exceptional values, Proc. Amer. Math. Soc. 38 (1973), 75-79.

3. P. Montel, Leçons sur les familles normales de fonctions analytiques et leurs applications, Gauthier-Villars, Paris, 1927.

4. P. C. Rosenbloom, The fix-points of entire functions, Medd. Lunds Univ. Mat. Sem. Tome Suppl. (1952), 186-192. MR 14, 546.

Department of Mathematics, University of Nebraska, Lincoln, Nebraska 68508 